Marquette University

e-Publications@Marquette

$5-25-2019$

\title{
Examining Latino Family Participation in Treatment for Childhood ADHD: The Role of Parental Cultural Factors and Perceptions
}

Theresa Lauer Kapke

Marquette University

Margaret A. Grace

Marquette University

Al Castro

United Community Center

Alyson C. Gerdes

Marquette University, alyson.gerdes@marquette.edu

Follow this and additional works at: https://epublications.marquette.edu/psych_fac

Part of the Psychology Commons

\section{Recommended Citation}

Kapke, Theresa Lauer; Grace, Margaret A.; Castro, Al; and Gerdes, Alyson C., "Examining Latino Family Participation in Treatment for Childhood ADHD: The Role of Parental Cultural Factors and Perceptions" (2019). Psychology Faculty Research and Publications. 430.

https://epublications.marquette.edu/psych_fac/430 


\section{Marquette University}

\section{e-Publications@Marquette}

\section{Pscyhology Faculty Research and Publications/College of Arts and Sciences}

This paper is NOT THE PUBLISHED VERSION; but the author's final, peer-reviewed manuscript. The published version may be accessed by following the link in th citation below.

Child \& Family Behavior Therapy, Vol. 41, No. 2 (2019): 84-109. DOI. This article is (C) Taylor \& Francis (Routledge) and permission has been granted for this version to appear in e-Publications@Marquette. Taylor \& Francis (Routledge) does not grant permission for this article to be further copied/distributed or hosted elsewhere without the express permission from Taylor \& Francis (Routledge).

\section{Examining Latino Family Participation in Treatment for Childhood ADHD: The Role of Parental Cultural Factors and Perceptions}

Theresa L. Kapke, PhD

Department of Psychology, Marquette University, Milwaukee, USA;

Margaret A. Grace, MS

Department of Psychology, Marquette University, Milwaukee, USA;

Al Castro, MS

United Community Center/Centro de la Comunidad Unida, Milwaukee, USA

Alyson C. Gerdes, PhD

United Community Center/Centro de la Comunidad Unida, Milwaukee, USA

\section{Abstract}

Attention-deficit/hyperactivity disorder (ADHD) is a common mental health disorder in childhood.

Unfortunately, Latino youth and their families are at increased risk of demonstrating poor treatment outcomes. The current study examined the impact of parental cultural factors and perceptions on Latino family 
participation in a psychosocial intervention for childhood ADHD. Sixty-one Latino youth and at least one of their primary parents and teachers participated in the current study. Results indicated that parental acculturation, attitudes regarding treatment, and baseline severity of child symptomatology and functional impairment were related to treatment participation outcomes. Implications and future directions are discussed.

\section{Keywords}

Acculturation, ADHD, child psychology, Latino/a, stigma

\section{Introduction}

Attention-deficit/hyperactivity disorder (ADHD) is one of the most com-mon mental health disorders in childhood, affecting approximately $5 \%$ of children in the United States (American Psychiatric Association [APA], 2013). ADHD is thought to consist of developmentally inappropriate levels of inattention, hyperactivity, and/or impulsivity, frequently leading to significant functional impairment across various settings and other psychosocial difficulties (APA, 2013). Although efficacious treatments for childhood ADHD have been identified, including pharmacological and psychosocial treatments (i.e., parent management training [PMT] and class-room behavior management interventions; Corkum, Bessey, McGonnell, \& Dorbeck, 2015; Pelham \& Fabiano, 2008), available research suggests that the majority of children and families who require mental health services do not receive them (Kataoka, Zhang, \& Wells, 2002).

Despite comparable prevalance rates of ADHD (Bird et al., 2008), ethnic minority populations, including Latino youth and their families, are less likely than European American youth to receive diagnoses and ADHD treatment (Eiraldi \& Diaz, 2010; Morgan, Hillemeier, Farkas, \& Maczuga, 2014). Various practical and sociocultural factors are thought to contribute to the underdiagnosis of ADHD in Latino youth. Some of the barriers that have been linked to decreased likelihood of mental health care utilization for Latino youth, ultimately leading to fewer opportunities for diagnosis and treatment, include access and availability of culturally sensitive and linguistically appropriate mental health care services; lack of transportation, childcare, insurance, and experience with the mental health care system in the United States; scheduling difficulties; cost; and potential concerns regarding the impact of mental health care utilization on immigration status (see Gerdes, Lawton, Haack, \& Hurtado, 2013; Kapke \& Gerdes, 2015 for a review). In addition, research suggests that an overreliance on parents' reports of symptomatology, which may be subject to cultural biases and differences in etiological beliefs; concerns regarding seeking help outside the family unit; poorer quality of care, communication, and collaboration with health care providers; and providers' biases against diagnosing children from racial/ethnic minority backgrounds with conditions requiring treatment (see Gerdes et al., 2013; Kapke \& Gerdes, 2015; Morgan et al., 2014; Paidpati, Brawner, Eiraldi, \& Deatrick, 2017 for a review) also likely contribute to existing disparities.

These findings are alarming, especially since the Latino population is the largest and most rapidly growing ethnic minority group in the United States (Pew Hispanic Center, 2011). Although research suggests that Latino families may be more receptive to psychosocial treatments as opposed to medication, these services are underutilized as well (Arcia, Fernandez, \& Jaquez, 2004; Bauermeister et al., 2003; Eiraldi \& Diaz, 2010). Despite the welldocumented low rates of ADHD service use, it is important to note that Latino youth and their families should not be viewed as resistant to or unable to benefit from treatment. Rather, attention should be paid to the factors that influence accessibility and quality of services, including specific barriers to treatment and the need for culturally responsive interventions (Eiraldi \& Diaz, 2010; (Miranda et al., 2002).

\section{Overview of Latino Family Participation in Child Mental Health Services}

Health care utilization is thought to consist of both the identification and receipt of services, and although many Latino families also may seek sup-port from informal sources (Cauce et al., 2002; Garland et al., 2005), the current study focuses on Latino family participation in formal mental health services and the receipt of services once they have sought help. Unfortunately, research suggests that Latino youth and families are at risk of 
demonstrating poor treatment outcomes, including treatment attendance, retention, engagement, and response. In the general population, it is estimated that up to $60 \%$ of youth and families drop out of treatment before the initial intake appointment (Gopalan et al., 2010), and approximately $30 \%$ to $75 \%$ of youth and families drop of out treatment before receiving the prescribed intervention (de Haan, Boon, de Jong, Hoeve, \& Vermeiren, 2013). Although there is limited research on treatment retention for Latino families, ethnic minority youth and families, especially those from low-socioeconomic-status (SES) backgrounds, demonstrate poorer engagement in child therapy services than European American youth (Gopalan et al., 2010; Ingoldsby, 2010), leading to reduced treatment effectiveness (Barrett et al., 2008). Although SES factors and parental stress likely contribute to these findings (Nix, Bierman, \& McMahon, 2009; Reyno \& McGrath, 2006), cultural and family system factors also are theorized to impact family engagement in PMT (Forehand \& Kotchick, 1996, 2002). Parental cultural factors and perceptions are of particular interest in the current study, given parents' unique role in initiating and sustaining treatment.

\section{Factors that Influence Latino Family Participation in Child Mental Health}

\section{Services}

Various factors have been found to predict participation in ADHD treatment in the general population, such that females, younger children, and those with lesser severity of symptoms and higher SES demonstrate improved treatment adherence and response (Corkum et al., 2015; Reyno \& McGrath, 2006). Research on the impact of comorbidity on ADHD treatment outcomes has presented mixed findings (e.g., Graetz, Sawyer, Baghurst, \& Hirte, 2006; Thiruchelvam, Charach, \& Schachar, 2001). It is unclear how these findings extend to Latino families in the United States, especially considering their unique health care accessibility and utilization needs. In addition, much of the available literature centers on pharmaco-logical treatment outcomes and fails to distinguish between the different aspects of treatment participation (Corkum et al., 2015). Thus, more research is needed to understand the factors that impact Latino family participation in psychosocial treatments for ADHD.

\section{Parental Cultural Factors: Acculturation and Ethnic Identity}

Given that nearly $40 \%$ of Latino individuals in the United States are foreign-born (U.S. Census Bureau, 2014) and over half of Latino youth in the U.S. are children of immigrant parents (Pew Hispanic Center, 2009), cul-tural factors must be considered when examining Latino family participation in mental health treatment.

Acculturation, one of the major cultural concepts that has been identified, is defined as the multidimensional process that occurs when individuals from different cultural backgrounds interact with each other, which often produces changes in one's behavior, beliefs, and values related to their ethnic culture of origin and/or host culture (Martın, 1992; Schwartz, Unger, Zamboanga, \& Szapocznik, 2010). Most studies have found that greater orientation to U.S. mainstream culture is associated with increased service use (Lara, Gamboa, Kahramanian, Morales, \& Bautista, 2005). Proxy measures of acculturation, such as time in the United States and English language use, also have been associated with a higher degree of mental health service use (Keyes et al., 2012; Nandi et al., 2008), yet the few existing studies examining Latino caregiver acculturation status and youth mental health service utilization present mixed findings (e.g., Ho, Yeh, McCabe, \& Hough, 2007; Kim, Lau, \& Chorpita, 2016; McCabe, 2002a).

Ethnic identity is another cultural factor that has been linked to participation in mental health services and refers to the sense of identification with and belonging to one's ethnic community and culture of origin (Phinney \& Ong, 2007). Although ethnic identity has been linked to various psychological benefits for Latino individuals (Quintana \& Scull, 2009), it may have differential effects on service utilization. For example, a recent study found that individuals reporting strong Latino ethnic identity were less likely to use mental health services for mood and anxiety disorders, even after controlling for severity of symptoms, income, education, and insurance (Keyes et al., 2012). Thus, research suggests that both acculturation and ethnic identity should be taken into account when examining Latino family participation in ADHD treatment. 


\section{Parental Perceptions: Attitudes and Expectations Regarding Treatment}

In addition to parental cultural factors, parental perceptions regarding mental health and treatment also must be examined. Researchers suggest that parental cognitions impact family participation in ADHD treatment, such as ideas and beliefs regarding ADHD, parental efficacy, and perceptions regarding child behavior and mental health treatment (Hoza, Johnston, Pillow, \& Ascough, 2006). Stigmatized attitudes among parents have been identified as a major barrier to ADHD service utilization (Corkum et al., 2015). Stigma may be particularly salient for Latino families, as studies indicate that ethnic minority individuals are likely to endorse mental health stigma, negative attitudes towards health care pro-viders, and a belief that problems should be handled within the family (see Villatoro, Morales, \& Mays, 2014 for a review). Research suggests that care-givers from ethnicminority backgrounds may hesitate to seek treatment

for their child's behavioral issues due to feelings of self-blame and perceived stigma from their communities, which may lead to decreased perceived competence and social interactions (Fernandez \& Arcia, 2004).

Expectations regarding treatment also may have implications for family participation. Research suggests that many Latino families may experience low health literacy (Gazmararian, Curran, Parker, Bernhardt, \& DeBuono, 2005) and have limited knowledge, resources, and experience related to use of formal mental health services in the United States, all of which may influence parental expectations regarding treatment, especially if parental expectations are incongruent with those of the health care provider (McCabe, 2002a). Other systemic barriers related to receipt of ADHD treatment also have been identified, such as parental perceptions of financial burden of treatment, lack of time, lack of insurance, and long waiting lists (Corkum et al., 2015), but less is known regarding Latino families specifically.

\section{Current Study}

Although existing research provides a good foundation for understanding barriers related to child mental health services in the United States, information related to the development and progress of ADHD treatment among Latino youth is scarce, and little is known about psychosocial treatment of ADHD in Latino families (Eiraldi \& Diaz, 2010; Gerdes, Kapke, Lawton, Grace, \& Hurtado, 2015). Improved understanding of the factors that impact Latino family participation in mental health services is crucial to addressing existing mental health disparities. The current study aimed to contribute to this area of research by providing knowledge about the way in which parental cultural factors (i.e., acculturation and ethnic identity) and perceptions (i.e., attitudes and expectations regarding treatment) impact Latino family participation in a psychosocial intervention for child-hood ADHD, including attendance, retention, engagement, and treatment response outcomes.

It was hypothesized that after accounting for treatment condition and child demographic/diagnostic factors (i.e., child sex, age, severity of ADHD symptoms, comorbidity, and SES), greater orientation to Latino culture on measures of acculturation and higher levels of ethnic identity among parents would predict poorer treatment participation outcomes, including attendance, retention, engagement, and treatment response. However, greater orientation to U.S. mainstream culture on measures of acculturation and lower levels of ethnic identity among parents were expected to predict improved treatment participation outcomes. Similarly, it was hypothesized that greater endorsement of specific parental attitudes and expectations regarding treatment (i.e., belief that problems should be handled within the family unit, stigmatized attitudes related to mental health treatment, perceived barriers to treatment, and expectations for a speedy recovery in treatment) would predict poorer treatment participation outcomes.

\section{Method}

\section{Participants}

Based on multicultural guidelines for promoting participation of Latino families in research (e.g., National Institutes of Health, 2002; Yancey, Ortega, \& Kumanyika, 2006), partnerships were established with local schools 
and a community center predominantly serving Latino families in an urban setting, and in-person recruitment took place with families, teachers, and school staff during various school-sponsored events, as well as via school announcements, mailings, and word-of-mouth referral. Partnerships also were established with health care providers from a local community health center in order to identify eligible families.

Participants for the current study included Latino youth between the ages of 5 and 13 years who were assessed for ADHD as part of a larger research project (see Gerdes, Kapke, Grace, \& Castro, 2017) and at least one of their primary parents and teachers. Sixty-one families participated in the current study, including 61 Latino youth (44 males, 17 females) with a mean age of 7.98 years ( $S D=2.57), 60$ mothers and 26 fathers with a mean age of 37.26 years $(S D=7.29)$, and 61 teachers. Of the 86 participating parents, 38 parents identified as Spanish speaking only, and 48 parents identified as speaking both Spanish and English, including being bilingual, primarily speaking Spanish and some English, and/or primarily speaking English and some Spanish.

\section{Procedure}

Interested families contacted the study team, after which an initial phone screen was conducted. The participating parent(s) and child had to self-identify as Latino. In addition, the parent(s) had to be Spanish speaking, although not necessarily Spanish speaking only. Participating children had to be 5 to 13 years old and display symptoms and functional problems consistent with ADHD. Children with existing diagnoses of intellectual disability, autism spectrum disorder, and/or a psychotic disorder were excluded from the study. Once eligibility was determined, a comprehensive, multi-modal, multi-informant ADHD assessment was conducted at a university-based clinic or local community center, depending on the family's preference. The ADHD assessment took approximately four hours to complete. The parent component was completed in Spanish; the child component was completed in English or Spanish, depending on the child's language preference; and the teacher component was completed in English.

After obtaining informed consent and assent from the participating parent(s) and child, the graduate clinician completed an unstructured inter-view with the parent(s) in order to gather background information and identify home-based treatment goals. The graduate clinician conducted the Disruptive Behavior Disorders (DBD) Structured Interview with the parent(s). The parent(s) also completed a series of questionnaires assessing ADHD symptomatology and functional impairment, demographic information, and parental factors, and the child completed self-report measures of anxiety and depression. Measures relevant to the current study are described below. All of the parent questionnaires were completed in Spanish, and assistance from bilingual graduate research assistants was available upon request. As part of the assessment, the graduate clinician obtained the contact information for the child's primary teacher in order to arrange a teacher meeting. After obtaining teacher consent, an unstructured interview was conducted in order to identify school-based treatment goals, and the teacher completed questionnaires assessing ADHD symptomatology and functional impairment.

If the child met criteria for ADHD based on the comprehensive assessment, the child's family was eligible to participate in the second phase of the study, which included a psychosocial intervention designed for treating ADHD in school-aged children. Families were randomly assigned to participate in one of two treatment groups, including standard treatment (ST) and a culturally adapted treatment (see Gerdes et al., 2015 for more detail on the culturally adapted treatment). Both treatments included eight PMT classes and a school-based intervention in the form of a Daily Report Card (DRC). The adaptations that were made centered on addressing practical barriers to treatment (e.g., child care, transportation, scheduling) and promoting the use of a more culturally congruent treatment for participating youth and their families. Ultimately, these adaptations aimed to improve family attendance, retention, engagement, and response. Various general adaptations were made to the assessment, feedback, and treatment phases, as well as specific treatment sessions. Also, two of the existing treatment sessions were replaced with content that was determined to be more consistent with cultural values and practical considerations (see Gerdes et al., 2015 for more detail). Classes were co-led by a graduate student 
clinician and a social worker from the local community center. The participating parent(s) attended weekly group-based PMT sessions, which took place for approximately two hours/week, as well as meetings at the school to discuss the DRC intervention with the child's primary teacher. Families participating in the culturally adapted treatment also received two 30-minute home visits, one of the adaptations that was made. At the end of treatment, the participating parent(s) and teacher completed questionnaires assessing ADHD symptomatology and functional impairment. Families received a $\$ 100$ gift card for completion of measures at pre- and posttreatment. Teachers received a $\$ 5$ gift card for completion of measures at pre-and posttreatment.

\section{Measures}

DBD Rating Scale

The DBD Rating Scale (Spanish translation by Gerdes et al., 2013; Pelham, Gnagy, Greenslade, \& Milich, 1992) is a 45-item parent- and teacher-report measure that was used to assess ADHD symptomatology. Responses are based a four-point scale, ranging from 0 (not at all present) to 3 (very much present), with higher scores indicate greater symptomatology. The English and Spanish versions of the DBD Rating Scale have demonstrated good reliability (Gerdes et al., 2013; Pelham, Fabiano, \& Massetti, 2005). Pretreatment mean scores for total ADHD symptoms (i.e., inattention and hyperactive/impulsivity) were utilized from both parent and teacher versions of the DBD to assess symptom severity at baseline, and posttreatment parent- and teacher-reported mean scores for total ADHD symptoms were utilized to assess symptomatology posttreatment. Pretreatment responses include ratings of the child's unmedicated behavior, and posttreatment responses reflect either medicated or unmedicated ratings, depending on child's ADHD medication status. Good internal consistency was found in the current study for both parent and teacher reports, including Cronbach's alphas of .94 and .86, respectively.

\section{ADHD-FX Scale}

The ADHD-FX Scale (Haack, Gerdes, Lawton, \& Schneider, 2016) is a 32-item parent- and teacher-report measure that was used to assess ADHD functional impairment across settings. Using a four-point scale that ranges from 0 (no effect)to3(a lot of effect), the ADHD-FX Scale assesses the extent to which functional problems affect the child in his/her daily life, with higher scores representing greater levels of functional impairment. Both English and Spanish versions of the scale have demonstrated good reliability and consistency (Haack, Gonring, Harris, Gerdes, \& Pfiffner, 2019). Mean scores for functional impairment at home, school, and with peers may be computed, as well as a total functional impairment score. Parent report of functional impairment at home and teacher report of functional impairment at school were used in the current study, as each reflects what the parent and teacher are likely to observe in their respective settings. Pretreatment scores were utilized to assess severity in child's functional impairment at baseline, and posttreatment scores were utilized to assess functional impairment posttreatment. Pretreatment responses include ratings of the child's unmedicated behavior, and posttreatment responses reflect either medicated or unmedicated ratings, depending on the child's ADHD medication status. Good internal consistency was found in the cur-rent study for both parent and teacher reports, including Cronbach's alphas of .93 and .92 , respectively.

\section{Acculturation Rating Scale for Mexican Americans (ARSMA-II)}

The ARSMA-II (Cuellar, Arnold, \& Maldonado, 1995) is a 30-item self-report measure that was used to assess parents' behavioral acculturation. Using a five-point scale that ranges from 1 (not at all)to 5 (extremely or almost always), the ARSMA-II largely assesses behavioral aspects of acculturation, including language preference and customs. The ARSMA-II can be used to compute mean scores for the Latino Orientation Scale (MOS) and the Anglo Orientation Scale (AOS), with higher scores indicating greater orientation to the respective culture. The current study modified the ARSMA-II by substituting "Latino" for "Mexican" or "Mexican American," which has been done in other studies (Steidel \& Contreras, 2003). The ARSMA-II has demonstrated good internal and testretest reliabilities (Cuellar et al., 1995). In the current study, good internal consistency was found for the MOS and AOS among mothers (Cronbach's alphas $=.82$ and .91 , respectively) and fathers (Cronbach's alphas $=.83$ and .92 , respectively). 
Mexican American Values Scale for Adolescents and Adult (MACVS)

The MACVS (Knight et al., 2010) is a 50-item self-report measure that was used to assess parents' cognitive acculturation. Using a five-point scale that ranges from 1 (not at all)to5 (completely), the MACVS assesses values that are frequently associated with Latino and U.S. mainstream culture, such as familism, respect, traditional gender roles, material success, and personal achievement. The MACVS can be used to compute mean scores for the Mexican American Values Scale (MAV) and the Mainstream Values Scale (AV), with higher scores indicate greater endorsement of cultural values. The MAV and AV have demonstrated good internal consistency (Knight et al., 2010). In the current study, good internal consistency was found for the MAV and AV for mothers (Cronbach's alphas $=.88$ and .70 , respectively) and fathers (Cronbach's alphas $=.89$ and .73 , respectively).

\section{Multigroup Ethnic Identity Measure-Revised (MEIM-R)}

The MEIM-R (Phinney \& Ong, 2007) is a six-item self-report measure that was used to measure parents' ethnic identity. Using a four-point scale that ranges from 1 (strongly disagree)to 4 (strongly agree), the MEIM-R assesses aspects of one's ethnic identity, including exploration and commitment. Mean scores for the exploration and commitment subscales can be computed, as well as a total score of ethnic identity, which was used in the cur-rent study. Higher scores indicate greater levels of agreement with the statements related to one's ethnic identity. The MEIM-R has demonstrated good reliability in past research (Phinney \& Ong, 2007). Good internal consistency was found in the current study for both mother and father reports, including Cronbach's alphas of .85 and .85 , respectively.

\section{Therapy Attitude Questionnaire (TAQ)}

The TAQ (McCabe, 2002b) is a self-report measure that was used to assess parents' attitudes regarding therapy. The TAQ can be used to compute mean scores for four constructs, including family/self-reliance, guilt feelings, reliance on discipline, and stigma. Using a five-point scale that ranges from 1 (strongly disagree)to5(strongly agree), higher scores indicate greater levels of agreement. The current study utilized the four-item family/selfreliance and five-item stigma scales, which reflect the beliefs that child-ren's problems do not require outside help and that seeking child mental health services is shameful. These scales have demonstrated good reliability in past research (McCabe, 2002a). In the current study, good internal consistency was found for the family/selfreliance and stigma scales among mothers (Cronbach's alphas $=.75$ and .71 , respectively) and fathers (Cronbach's alphas $=.72$ and .75 , respectively).

\section{Therapy Expectations Questionnaire (TEQ)}

The TEQ (McCabe, 2002c) is a self-report measure that was used to assess parents' expectation regarding therapy. The TEQ can be used to compute mean scores for three constructs, including directiveness, perceived barriers to therapy, and speed of recovery. Using a five-point scale that ranges from 1(strongly disagree) to 5 (strongly agree), higher scores indicate greater levels of agreement. The current study utilized the nine-item perceived barriers and the two-item speed of recovery scales. These scales have demonstrated adequate reliability in past research (McCabe, 2002a). In the current study, good internal consistency was found for the barriers and speed of recovery scales among mothers (Cronbach's alpha $=.84$ and $r=.78, p \leq .001$, respectively) and fathers (Cronbach's alpha $=.80$ and $r=.74, p \leq .001$, respectively).

\section{Demographic Questionnaire}

A demographic questionnaire was administered to parents to collect demo-graphic information about each participating parent and child, including age, sex, and socioeconomic status (SES). SES was assessed using the Hollingshead Four Factor Index of Social Status (Hollingshead, 1975), which considers one's marital status, employment status, education, and occupation in determining SES. 


\section{Outcome Variables}

Treatment Attendance and Retention

Attendance was measured according to number of PMT sessions attended by parents. Treatment retention was defined as family completion of the last scheduled session/class.

\section{Treatment Engagement}

Engagement was assessed by (1) homework completion and (2) therapist ratings of parental engagement. A total mean percentage of completion on various homework items was calculated for each child. Both therapists also rated the participating parent(s)'s level of engagement at the end of treatment on a five-point scale of 1 (very low)to 5 (very high), and a mean therapist rating engagement score for participating parent(s) was then computed for each family.

\section{Treatment Response}

Treatment response was assessed by (1) posttreatment ratings of symptomatology and functional impairment, (2) therapist ratings of treatment response, and (3) progress toward treatment goals. Posttreatment scores on the DBD Rating Scale and the ADHD-FX Scale were used to assess symptomatology and functioning impairment following treatment after control-ling for pretreatment levels. Both therapists also rated the participating child's improvement related to behavior and functioning and the participating parent(s)'s improvement related to parent/family functioning at the end of treatment on a five-point scale of 1 (none)to 5(substantial gains), and a mean treatment response score was computed for each participating child and parent(s). Finally, approximately five treatment goals were established for each participating child in the home and school settings, and the percentage of treatment goals that were met at the end of treatment was calculated for each child.

\section{Results}

\section{Preliminary Results}

Based on current guidelines for managing multisource data (Holmbeck, Li, Schurman, Friedman, \& Coakley, 2002), correlations between parent- and teacher-reported ADHD symptoms (DBD Rating Scale) were conducted. Given that pre- and posttreatment reports were not highly correlated ( $r=.12, n s ; r=.45, n s$, respectively), they were examined separately for all analyses.

\section{Predictor Variables and Treatment Participation Outcomes}

Independent-samples $t$ tests were conducted to examine mean differences in attendance, engagement, and treatment response outcomes according to treatment type, child sex, and comorbidity. No significant differences emerged according to child sex. Results indicated that families participating in the culturally adapted treatment demonstrated significantly higher homework completion and therapist-reported improvements in child and parent/family functioning, $M=88.83, S D=13.63 ; M=4.27, S D=0.55 ; M=4.31, S D=0.60$, respectively, compared to those in $S T, M=68.95, S D=24.36 ; t(45.22)=-3.92, p \leq .001 ; M=3.70, S D=1.12 ; t(41.73)=-2.54, p$ $\leq .05 ; \mathrm{M}=3.83, \mathrm{SD}=1.15 ; \mathrm{t}(43.32)=-2.00, \mathrm{p} \leq .05$, respectively. The effect sizes of these differences were medium to large. In addition, youth with comorbid diagnoses demonstrated significantly higher parent-reported ADHD symptomatology and functional impairment in the home setting posttreatment, $M=1.53, S D=0.70 ; M=$ $0.98, \mathrm{SD}=0.51$, respectively, compared to those without comorbid diagnoses, $\mathrm{M}=1.16, \mathrm{SD}=0.51 ; \mathrm{t}(56)=-2.29$, $\mathrm{p} \leq .05 ; \mathrm{M}=0.62, \mathrm{SD}=0.36 ; \mathrm{t}(56)=-3.09, \mathrm{p} \leq .01$, respectively. Effect sizes of these differences were medium to large.

Correlational analyses were then conducted to examine relations between the other child demographic/diagnostic variables (i.e., child age, SES, and baseline severity of ADHD symptoms and functional impairment) and parental cultural variables and perceptions regarding treatment with attendance, engagement, 
and treatment response outcomes. In summary, attendance and engagement outcomes (i.e., homework completion, therapist-rated parental engagement) were significantly related to father acculturation status. In addition, treatment response outcomes (i.e., posttreatment parent- and teacher-reported ratings of symptomatology and functional impairment) were significantly related to baseline severity of ADHD symptoms and functional impairment, parent acculturation status, and mothers' attitudes toward treatment (see Table 2).

Table 1. Parent, Family, and Child Demographics.

\begin{tabular}{|c|c|c|c|}
\hline Parent and Family Factors & & Child Factors & \\
\hline Age, M (SD) & $37.26(7.29)$ & Age, M (SD) & $7.98(2.57)$ \\
\hline Sex, n (\%) & & Sex, $n(\%)$ & \\
\hline Female & $60(69.77)$ & Female & $17(27.87)$ \\
\hline Male & $26(30.23)$ & Male & $44(72.13)$ \\
\hline Education, $n(\%)^{+}$ & & Grade level, n(\%) & \\
\hline Some high school or less & $52(60.47)$ & Kindergarten & $13(21.31)$ \\
\hline Graduated high school/GED & $17(19.77)$ & Elementary $\left(1^{\text {st }}-5^{\text {th }}\right.$ grade $)$ & $36(59.02)$ \\
\hline Some college & $11(12.79)$ & Middle school $\left(6^{\text {th }}-8^{\text {th }}\right.$ grade $)$ & $12(19.67)$ \\
\hline College or graduate degree & $4(4.65)$ & ADHD subtype, $n(\%)$ & \\
\hline Ethnicity, $n(\%)$ & & Inattentive only & $26(42.62)$ \\
\hline Latino, Mexican descent & $76(88.37)$ & Hyperactive/impulsive only & $7(11.48)$ \\
\hline Latino, Puerto Rican descent & $4(4.65)$ & Combined. & $28(45.90)$ \\
\hline Latino, other descent & $6(6.98)$ & Comorbid diagnoses & \\
\hline Language, $n(\%)$ & & None & $43(70.49)$ \\
\hline Only Spanish & $38(44.19)$ & ODD/CD & $14(22.95)$ \\
\hline Primarily Spanish, some English & $29(33.72)$ & Mood/anxiety & $4(6.56)$ \\
\hline Primarily, some Spanish & $2(2.33)$ & Medication status, $\mathrm{n}(\%)$ & \\
\hline Bilingual & $17(19.77)$ & Medicated & $12(19.67)$ \\
\hline Time in U.S., $\mathrm{n}(\%)$ & & Unmedicated & $49(80.33)$ \\
\hline $1-5$ years & $1(1.16)$ & Type of treatment, $\mathrm{n}(\%)$ & \\
\hline $6-10$ years & $17(19.77)$ & Standard treatment & $30(49.18)$ \\
\hline More than 10 years & $63(73.26)$ & Culturally adapted treatment & $31(50.82)$ \\
\hline Born in U.S. & $5(5.81)$ & Retention, $\mathrm{n}(\%)$ & \\
\hline Family structure n (\%) & & Yes & $58(95.08)$ \\
\hline Married/cohabitating parents & $42(68.85)$ & No & $3(4.92)$ \\
\hline Separated/divorced/widowed & $16(26.23)$ & Referral source, $\mathrm{n}(\%)$ & \\
\hline Single/never married & $3(4.92)$ & Health care provider referral & $13(21.31)$ \\
\hline \multirow[t]{2}{*}{ Family SES, M (SD) ${ }^{\mathrm{a}}$} & $23.43(11.13)$ & School referral & $26(42.62)$ \\
\hline & & Self-referral & $22(36.07)$ \\
\hline
\end{tabular}

Note. $\mathrm{N}=61$ families, including 61 youth, 60 mothers, and 26 fathers.

$\mathrm{TR}=$ therapist ratings, $\mathrm{TR}$ child $=$ therapist-rated child improvement, $\mathrm{TR}$ parent/family $=$ therapist-rated parent/ family improvement.

+ Indicates missing data for some participants.

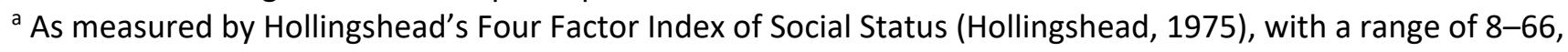
higher values indicating higher SES. 
Table 2. Results of Correlation Analyses Examining Child Sociodemographic/Diagnostic Variables and Parental Cultural Factors and Perceptions with Attendance, Engagement, and Treatment Response Outcomes.

\begin{tabular}{|c|c|c|c|c|c|c|c|c|c|c|c|}
\hline $\begin{array}{l}\text { Child and } \\
\text { Parent } \\
\text { Variables }\end{array}$ & $\begin{array}{c}\text { Attendance, Engagement, } \\
\text { and Treatment Response } \\
\text { Outcomes }\end{array}$ & & & & & & & & & & \\
\hline & $\mathrm{ATT}$ & $\begin{array}{l}\% \\
\text { HW }\end{array}$ & $\begin{array}{l}\text { TR } \\
\text { eng }\end{array}$ & $\begin{array}{l}\text { P2 } \\
\text { DBD }\end{array}$ & $\begin{array}{l}\text { T2 } \\
\text { DBD }\end{array}$ & $\begin{array}{l}\text { P2 FX } \\
\text { home }\end{array}$ & $\begin{array}{l}\text { T2 FX } \\
\text { school }\end{array}$ & $\begin{array}{l}\text { TR } \\
\text { child }\end{array}$ & $\begin{array}{l}\text { TR } \\
\text { parent/family }\end{array}$ & $\begin{array}{l}\% \\
\text { Home }\end{array}$ & $\begin{array}{l}\% \\
\text { School }\end{array}$ \\
\hline Child age & .04 & -.05 & -.06 & -.09 & -.03 & -.003 & .01 & -.14 & -.08 & $.22^{+}$ & -.04 \\
\hline Family SES & -.09 & -.15 & -.11 & .03 & .06 & -.18 & .04 & -.07 & -.07 & -.16 & -.10 \\
\hline P1 DBD & .05 & .19 & .13 & $.49 * * *$ & -.18 & $.40 * *$ & -.09 & .05 & .14 & .13 & .14 \\
\hline T1 DBD & -.19 & -.14 & .07 & .14 & $.55 * * *$ & -.18 & $.32 *$ & .003 & .07 & .004 & -.01 \\
\hline P1 FX home & .16 & $.25^{+}$ & .19 & $.40 * *$ & $-.29 *$ & $.55 * * *$ & -.12 & .01 & .11 & .15 & .18 \\
\hline T1 FX school & -.01 & .04 & .07 & .01 & $.34 * *$ & -.04 & $.41 * * *$ & .05 & .13 & .003 & -.13 \\
\hline $\begin{array}{l}\text { Mother } \\
\text { ARSMA-II MOS }\end{array}$ & .01 & -.04 & -.03 & $.23^{+}$ & -.11 & .09 & -.16 & -.09 & .03 & $-.22^{+}$ & -.08 \\
\hline $\begin{array}{l}\text { Mother } \\
\text { ARSMA-II AOS }\end{array}$ & -.12 & -.07 & $-.25^{+}$ & $-.27 *$ & .19 & -.10 & .16 & -.09 & -.13 & -.17 & -.11 \\
\hline $\begin{array}{l}\text { Mother } \\
\text { MACVS MAV }\end{array}$ & -.07 & .02 & .14 & -.03 & $.28_{-}^{*}$ & .15 & .18 & $.21^{+}$ & .11 & .11 & -.12 \\
\hline $\begin{array}{l}\text { Mother } \\
\text { MACVS AV }\end{array}$ & .09 & .11 & .03 & .06 & -.03 & .10 & .02 & .04 & -.01 & -.04 & -.10 \\
\hline $\begin{array}{l}\text { Mother MEIM- } \\
\text { R total }\end{array}$ & -.06 & -.03 & -.07 & .19 & -.001 & .18 & .01 & -.05 & -.02 & -.18 & .07 \\
\hline $\begin{array}{l}\text { Mother TAQ } \\
\text { fsr }\end{array}$ & -.04 & -.11 & .03 & -.02 & .18 & .01 & $.28_{-}^{*}$ & -.02 & -.18 & -.01 & $-.21^{+}$ \\
\hline $\begin{array}{l}\text { Mother TAQ } \\
\text { stigma }\end{array}$ & $.22^{+}$ & $.22^{+}$ & .07 & -.10 & -.01 & -.04 & $.26_{-}^{*}$ & .09 & .08 & .03 & .09 \\
\hline $\begin{array}{l}\text { Mother TEQ } \\
\text { barr }\end{array}$ & .14 & .13 & .02 & -.21 & -.14 & -.13 & -.04 & .04 & .01 & -.12 & .03 \\
\hline Mother TEQ sr & -.02 & .09 & -.03 & -.07 & .03 & -.01 & -.05 & -.01 & -.02 & .03 & -.12 \\
\hline $\begin{array}{l}\text { Father ARSMA- } \\
\text { II MOS }\end{array}$ & .01 & .03 & .12 & .29 & .01 & .14 & -.04 & .04 & .28 & -.13 & .06 \\
\hline $\begin{array}{l}\text { Father ARSMA- } \\
\text { II AOS }\end{array}$ & .25 & .25 & .16 & $-.39 *$ & -.09 & -.32 & -.09 & .26 & .06 & -.03 & -.20 \\
\hline
\end{tabular}




\begin{tabular}{|c|c|c|c|c|c|c|c|c|c|c|c|}
\hline $\begin{array}{l}\text { Father MACVS } \\
\text { MAV }\end{array}$ & .19 & .14 & .13 & -.15 & -.20 & .26 & .20 & .07 & .14 & .05 & .06 \\
\hline $\begin{array}{l}\text { Father MACVS } \\
\text { AV }\end{array}$ & $.48 * *$ & $.39 *$ & $.40_{-}^{*}$ & .16 & -.27 & .19 & -.19 & .24 & $.37^{+}$ & .14 & .05 \\
\hline $\begin{array}{l}\text { Father MEIM-R } \\
\text { total }\end{array}$ & .001 & -.13 & -.16 & -.22 & .12 & -.20 & .03 & -.12 & -.16 & -.16 & .001 \\
\hline Father TAQ fsr & .26 & .20 & .07 & -.30 & -.19 & -.20 & -.14 & .20 & .21 & .22 & -.23 \\
\hline $\begin{array}{l}\text { Father TAQ } \\
\text { stigma }\end{array}$ & .06 & -.12 & -.14 & -.26 & -.24 & -.27 & -.19 & -.04 & -.13 & .27 & .08 \\
\hline $\begin{array}{l}\text { Father TEQ } \\
\text { barr }\end{array}$ & .25 & .18 & .07 & -.17 & -.25 & -.18 & -.18 & -.02 & .06 & .20 & .06 \\
\hline Father TEQ sr & .16 & .12 & -.12 & -.12 & -.28 & -.25 & -.22 & .08 & .05 & .08 & -.11 \\
\hline
\end{tabular}

Note. $\mathrm{ATT}=$ attendance; $\% \mathrm{HW}=\%$ homework completed; TR eng = therapist-reported parental engagement; P1/P2 DBD/FX home = pre-/posttreatment parent DBD ADHD mean/ADHD FX Scale home impairment; T1/T2 DBD/FX school = pre-/posttreatment teacher DBD ADHD mean/ADHD FX Scale school impairment; TR child = therapist-reported child improvement; TR parent/family = therapist-reported parent/family improvement; \% home/school = \% of home-/school-based goals achieved; MOS = ARSMA-II Latino Orientation Scale; AOS = ARSMA-II Anglo Orientation Scale; MAV = MACVS Mexican American Values Scale; AV = MACVS Mainstream Values Scale; MEIM-R total = MEIM-R total ethnic identity; TAQ fsr = TAQ family/self-reliance scale; TAQ stigma $=$ TAQ stigma scale; TEQ barr = TEQ barriers scale; TEQ $s r=$ TEQ speed of recovery scale.

${ }^{\mathrm{a}} \mathrm{n}=26-61$.

$* p \leq .05 ; * * p \leq .01 ; * * * p \leq .001 ;+p \leq .10$.

Independent-samples t tests were then conducted to examine differences in child demographic/diagnostic variables and parental cultural variables and perceptions regarding treatment according to retention status. Given the small sample size of fathers of families who were not retained in treatment ( $\mathrm{n}$ $=1$ ), only mothers' data were examined. Results indicated that mothers of families who dropped out prematurely demonstrated significantly higher orientation to U.S. mainstream culture on the behavioral measure of acculturation, $M=3.46, S D=1.3$, compared to those who were retained in treatment, $M=2.38, S D=0.82 ; t(58)=2.16, p \leq .05$. The effect size of this difference was large. Finally, chi-square tests for independence also were conducted to examine differences in treatment type, child sex, and comorbidity according to retention status. No significant differences were found.

\section{Primary Results}

Based on the significant independent variables that emerged from the preliminary analyses, follow-up hierarchical multiple regression analyses were conducted, all of which were interpreted at the last step that produced a significant change in $R^{2} .{ }^{1}$ Hierarchical multiple regressions were only conducted for outcome variables that were significantly correlated with more than one predictor variable. In order to examine posttreatment parent- and teacherreported ADHD symptomatology and functional impairment as treatment response outcomes, medicated or unmedicated pretreatment ratings were controlled for in step 1, depending on the child's medication status posttreatment 
Hypothesis 1: Parental Cultural Factors and Treatment Participation Outcomes

In order to test the first set of hypotheses related to parental cultural factors and attendance, retention, engagement, and treatment response outcomes, hierarchical multiple regression analysis was conducted for posttreatment parent-reported ADHD symptomatology (see Table 3). Pretreatment parent-reported ADHD symptomatology was entered at step 1, and mother or father ARSMA-II AOS results were entered into step 2. Results indicated that the mother and father acculturation factors entered at step 2 explained an additional $6.9 \%$ and $17.8 \%$ of the respective variances in ADHD symptomatology posttreatment, which were significant changes, $F(1,54)=5.49, p \leq .05 ; F(1,22)=6.89, p \leq .05$, respectively. The overall models were significant, $F(2$, $54)=12.84, p \leq .001 ; F(2,22)=8.35, p \leq .01$, and explained $32.2 \%$ and $43.1 \%$ of the respective total variances in ADHD symptomatology. The effect sizes of the respective final models were small to medium. In the model examining mother acculturation, examination of individual factors indicated that both pretreatment parentreported ADHD symptomatology and mother ARSMA-II AOS were significant predictors, $\beta=.50, p \leq .001$; $\beta=-.26, p \leq .05$; respectively. In the model examining father acculturation, examination of individual factors indicated that both pretreatment parent-reported ADHD symptomatology and father ARSMA-II AOS were significant predictors, $\beta=.53, p \leq .01 ; \beta=-.42, p \leq .05$; respectively. This suggests that increased parent-reported ADHD symptomatology at baseline was related to increased parent-reported ADHD symptomatology posttreatment, and greater orientation to U.S. mainstream culture on parents' behavioral measures of acculturation was related to decreased parent-reported ADHD symptomatology posttreatment.

Table 3. Summary of Hierarchical Multiple Regression Analyses for Parental Cultural Factors and Perceptions Regarding Treatment Predicting Treatment Response Outcomes.

\begin{tabular}{|c|c|c|c|c|c|c|}
\hline & P2 DBDa & & P2 DBD卢 & & T2 FX schools & \\
\hline & Step 1 & Step 2 & Step 1 & Step 2 & Step 1 & Step 2 \\
\hline Variables & $\beta$ & & $\beta$ & & $\beta$ & \\
\hline P1 DBD med/unmed & $.50 * *$ & $.50 * *$ & $.50 * *$ & $.53 * *$ & & \\
\hline T1 FX school med/unmed & & & & & $.61 * * *$ & $.56 * * *$ \\
\hline Mother ARSMA-II AOS & & $-.26 *$ & & & & \\
\hline Father ARSMA-II AOS & & & & $-.42 *$ & & \\
\hline Mother TAQ fsr & & & & & & $.22_{-}^{*}$ \\
\hline Mother TAQ stigma & & & & & & .11 \\
\hline$R^{2}$ & $.25 * * *$ & $.32 * * *$ & $.25 * *$ & $.43 * *$ & $.37 * * *$ & $.43 * * *$ \\
\hline$\Delta R^{2}$ & $.25 \overline{* * *}$ & $.07 *$ & $.25 \overline{* *}$ & $.18_{-}^{*}$ & $.37 \overline{* * *}$ & $.06_{-}^{*}$ \\
\hline$f^{2}$ & & .10 & & .31 & & .11 \\
\hline
\end{tabular}

Note. P1 DBD med/unmed = pretreatment parent DBD medicated/unmedicated ADHD mean; T1 FX school med/unmed = pretreatment teacher ADHD FX Scale medicated/unmedicated school impairment; AOS = ARSMAII Anglo Orientation Scale; TAQ fsr = TAQ family/self-reliance scale; TAQ stigma = TAQ stigma scale; P2 $\mathrm{DBD}=$ posttreatment parent ADHD mean; T2 FX school = posttreatment teacher ADHD FX Scale school impairment.

Based on Cohen's (1988) guidelines, $f^{2} \geq .02=$ small, $f^{2} \geq .15=$ medium, and $f^{2} \geq .35=$ large.

${ }^{\mathrm{a}} n=58$.

${ }^{\mathrm{b}} n=26$.

${ }^{\mathrm{c}} n=60$.

${ }^{*} p \leq .05 ;{ }^{* *} p \leq .01 ;{ }^{* * *} p \leq .001 ;{ }^{+} p \leq .10$.

Hypothesis 2: Parental Perceptions and Treatment Participation Outcomes

In order to test the second set of hypotheses related to parental attitudes and expectations regarding treatment and attendance, retention, engagement, and treatment response, hierarchical multiple regression analysis was 
conducted for posttreatment functional impairment in the school setting (see Table 3). Pretreatment functional impairment in the school setting was entered at step 1, and mother TAQ family/self-reliance (fsr) and stigma scales were entered at step 2 . The parental perceptions regarding treatment that were entered at step 2 explained an additional $6.4 \%$ of the variance in functional impairment in the school setting, which was a significant change, $F(2,56)=3.16, p \leq .05$. The overall model was significant, $F(3,56)=14.27, p \leq .001$, and explained $43.3 \%$ of the variance in functional impairment in the school setting. The effect size of the final model was small. Examination of individual factors indicated that both pretreatment teacher-reported functional impairment and mother TAQ fsr were significant predictors, $\beta=.56, p \leq .001 ; \beta=.22, p \leq .05$, respectively, suggesting that increased teacher-reported functional impairment in the school setting at baseline and greater endorsement of the belief that children's problems do not require outside help among mothers were related to higher levels of functional impairment in the school setting posttreatment.

\section{Discussion}

In order to improve current understanding of factors that impact Latino family participation in a psychosocial intervention for childhood ADHD, the goal of the current study was to examine the impact of parental cultural fac-tors and attitudes and expectations regarding treatment on Latino family participation in treatment, including attendance, retention, engagement, and treatment response outcomes, after controlling for the effects of significant treatment condition and child demographic/diagnostic variables. Research suggests that Latino youth and their families are especially unlikely to receive diagnoses and ADHD treatment (Eiraldi \& Diaz, 2010; Morgan et al., 2014). In addition, Latino youth and their families are at increased risk for premature termination when they seek treatment (McCabe, 2002a; Miranda, Azocar, Organista, Mu noz, \& Lieberman, 1996), leading to decreased treatment effectiveness (Barrett et al., 2008). Better understanding of the way in which parental cultural factors and perceptions influence Latino family participation in treatment will aid researchers and clinicians in working to mitigate these existing disparities and promote the use of more culturally responsive assessment and psychosocial treatment for childhood ADHD in Latino families (Eiraldi \& Diaz, 2010; Morgan et al., 2014).

\section{Parental Cultural Factors and Treatment Participation Outcomes}

The first prediction that greater orientation to Latino culture on behavioral and cognitive measures of acculturation and higher levels of ethnic identity among parents would predict poorer attendance, retention, engagement, and treatment response outcomes, whereas greater orientation to U.S. mainstream culture and lower levels of ethnic identity would predict improved treatment outcomes, was partially supported. Regarding treatment attendance and engagement, fathers' endorsement of U.S. mainstream values was related to higher levels of family attendance, homework completion, and therapist-reported parental engagement. These findings are sup-ported by previous literature documenting the relation between orientation to U.S. mainstream culture and other proxy measures of acculturation (e.g., greater length of time in the United States and English language preference) and increased service use (Keyes et al., 2012; Lara et al., 2005; Nandi et al., 2008). It is noteworthy that fathers' acculturation status, in particular, was related to family attendance and engagement in treatment, especially given the smaller percentage of participating fathers. Research suggests that acculturation is one of the many factors that impacts Latino fathers' involvement with their children, as more traditionally oriented fathers may be less likely to be involved in caregiving responsibilities (see Cabrera \& Bradley, 2012 for a review) or participate in child mental health services (McCabe, Yeh, Garland, Lau, \& Chavez, 2005). Given that Latino families have emphasized the need to involve fathers and extended family members in behavioral treatment (McCabe et al., 2005) and that father involvement in parent training has been linked to improved child outcomes (Lundahl, Tollefson, Risser, \& Lovejoy, 2008), better understanding of the factors that impact Latino father engagement is needed.

In contrast to these findings, results also indicated that mothers of families who dropped out of treatment prematurely demonstrated significantly higher levels of orientation to U.S. mainstream culture on the behavioral 
measure of acculturation than those retained in treatment. That said, it is impossible to draw conclusions from such a small number of families who failed to complete treatment ( $1 / 43)$, and the significance difference in sample size between the two groups that were examined must be considered when interpreting these results. However, this finding may point to the het-erogeneity that exists within the Latino population in the United States (Ennis, Rıos-Vargas, \& Albert, 2011; Martinez \& Villarruel, 2009). From a service utilization perspective, we may have expected these families to complete treatment successfully, yet the cultural characteristics of the current sample may have impacted these families' ability or desire to remain in treatment, including parents' strong behavioral orientation to traditional Latino culture. Thus, the families who dropped out of treatment prematurely may have fared better in a less traditionally oriented group of parents, particularly related to cultural practices and customs. These findings highlight the need to assess and consider parental acculturation status when identifying the optimal treatment for families participating in psycho-social treatment for childhood ADHD.

Regarding parental cultural factors and treatment response outcomes, greater orientation to U.S. mainstream culture on parents' behavioral measure of acculturation was related to decreased parent-reported ADHD symptomatology posttreatment. Similarly, results indicated that mothers' endorsement of traditional Latino values was related to increased teacher-reported symptomatology posttreatment. However, results indicated that both mother and father behavioral acculturation orientations and parent-reported ADHD symptomatology pretreatment proved to be the most salient predictors of parent-reported ADHD symptomatology posttreatment. These findings are consistent with existing literature documenting the relation between more severe child behavioral problems pretreatment and poorer treatment outcomes among children receiving mental health services (Corkum et al., 2015; Reyno \& McGrath, 2006), as well as research indicating that Latino individuals more closely oriented to U.S. mainstream society demonstrate better service utilization outcomes (Keyes et al., 2012; Nandi et al., 2008). Although the research on caregiver acculturation status has presented mixed findings (e.g., Ho et al., 2007; Kim et al., 2016; McCabe, 2002a), researchers emphasize the crucial need to examine parental cultural factors in the context of PMT in order to better understand parenting behaviors and service utilization outcomes for Latino families participating in child mental health services (Barker, Cook, \& Borrego, 2010; McCabe et al., 2005). It is noteworthy that the effect size for father behavioral acculturation status was larger than that for mothers, which may highlight the impact of father cultural factors in the context of Latino families participating in PMT for childhood ADHD.

The effects of acculturation and related cultural constructs are complex. Although adherence to one's ethnic culture of origin has been associated with various positive psychosocial outcomes for Latino individuals (Gonzales, Fabrett, \& Knight, 2009), it also presents challenges for service utilization. Similarly, adherence to U.S. mainstream culture has been linked to negative health and educational outcomes in research examining the "immigrant paradox," a pattern of findings in which increased length of time in the United States is associated with more psychosocial problems for Latino individuals (Garcia Coll \& Marks, 2012; Vega, Sribney, AguilarGaxiola, \& Kolody, 2004), but it also seems to help to aid health care ser-vice utilization (Keyes et al., 2012; Lara et al., 2005; Nandi et al., 2008). Given that more traditionally oriented families appear to be at increased risk of experiencing negative treatment outcomes, these findings support recent efforts to improve the way in which existing evidence-based treatments engage families from diverse sociocultural backgrounds, such as the use of linguistically and culturally adapted treatments and strategies to reduce the effects of environmental stressors and barriers to treatment (Barker et al., 2010; Calzada, 2010).

\section{Parental Perceptions and Treatment Participation Outcomes}

The second prediction that greater endorsement of specific parental attitudes and expectations regarding treatment (i.e., belief that problems should be handled within the family unit, stigmatized attitudes related to mental health treatment, perceived barriers to treatment, and expectations for a speedy recovery in treatment) would predict poorer levels of attendance, retention, engagement, and treatment response outcomes was partially supported. Results indicated that mothers' belief that children's problems should be handled within the 
family and higher levels of stigmatized attitudes were related to higher levels of teacher-reported functional impairment in the school setting posttreatment, indicating lesser improvement in functioning. However, both baseline functional impairment in the school setting and the belief that children's problems should be handled with the family unit proved to be the most salient predictors of teacher-reported functional impairment in the school setting posttreatment. These results are supported by previous work documenting the detrimental effects of severe child behavioral problems pretreatment (Corkum et al., 2015; Reyno \& McGrath, 2006) and mental health stigma on treatment outcomes among families participating in child mental health services (see Villatoro et al., 2014 for a review). These results seem to point to familism, a multi-dimensional cultural construct that centers on prioritizing family needs over individual desires, strong family relationships and interconnectedness, dependence on one's family, and strong family loyalty (Steidel \& Contreras, 2003), which may make Latino families less amenable to seeking or receiving formal mental health services. Thus, results suggest that it may be especially important for mental health care providers to take this cultural value into account and assess for stigmatized attitudes when conducting parent training with Latino families (Barker et al., 2010; Calzada, 2010).

\section{Limitations}

Several limitations should be noted. First, the current study included a fairly homogenous sample of Latino youth and their families, largely representing a more traditionally oriented, lower-SES community of MexicanAmerican families living in an urban setting. This likely contributed to the relatively limited variability found within measures examining parental cultural factors and perceptions regarding treatment, and caution is warranted in generalizing these findings to other Latino subgroups in other geographical areas. Future studies should aim to replicate these findings in a larger, more representative sample of Latino youth and their families, including a greater number of participating fathers and extended family members, as well as examine follow-up data. Finally, limitations in the measurement of parental engagement and improvements in child and parent/family functioning should be noted. Although the current study attempted to limit individual bias by combining treatment facilitators' ratings, the facilitators rated these constructs at the end of the intervention, and this may have contributed to retrospective bias in reporting. Thus, future studies should aim to rate engagement and improvement variables at multiple points throughout the course of treatment.

\section{Implications and Future Directions}

Despite these limitations, the current study has several important implications and presents some exciting areas for future research. First, these findings bring attention to the importance of assessing parental cultural factors and perceptions among Latino families participating in psychosocial treatment for childhood ADHD, including acculturation, stigmatized mental health attitudes, and the belief that children's problems should be managed within the family unit. In order to develop a better understanding of the various factors that likely contribute to Latino family participation and identify families who may be in need of additional support, future studies should aim to assess related constructs, such as endorsement of specific values related to both traditional Latino and U.S. mainstream cultures, acculturation stressors, perceived social support, parental stress, treatment acceptability, and therapeutic alliance. Also, given the significant linguistic and cultural variability that exists within the Latino population in the United States, results suggest that a one-size-fits-all treatment approach is not appropriate or possible. Thus, careful consideration should be made in determining the best treatment approach for families in order to optimize treatment outcomes, and future studies should aim to improve understanding of different Latino subgroups in the United States. Furthermore, findings from the current study demonstrate the importance of assessing acculturation from a multidimensional, bidirectional perspective, as cognitive and behavioral acculturation related to both cultures appear to demonstrate unique effects on Latino family participation in child mental health services. Thus, more research is needed to better understand the most effective ways of promoting attendance, retention, engagement, and treatment response outcomes, for which culturally adapted interventions may be appropriate and necessary. In particular, future studies should 
aim to examine the unique contributions of the adaptations that likely contributed to improved engagement (i.e., homework completion) and treatment response outcomes (i.e., therapist-reported improvements in child and parent/family functioning) among participants in the current study, such as more in vivo practice and discussion of barriers to use of skills in treatment sessions, increased access to materials and opportunities to practice the use of skills outside of treatment sessions, efforts to involve extended family members and other social supports as needed, and use of more culturally sensitive assessment and intervention strategies.

\section{Acknowledgments}

This research was supported by the Eunice Kennedy Shriver National Institute of Child Health and Human Development of the National Institutes if Health under Award Number R21HD078553. The content is solely the responsibility of the authors and does not necessarily represent the official views of the National Institutes of Health

\section{References}

American Psychiatric Association [APA]. (2013). Diagnostic and statistical manual of mental disorders (5th ed.). Washington, DC: American Psychiatric Publishing.

Arcia, E., Fernández, M. C., \& Jáquez, M. (2004). Latina mothers' stances on stimulant medication: Complexity, conflict, and compromise. Journal of Developmental and Behavioral Pediatrics, 25(5), 311-317.

Barker, C. H., Cook, K. L., \& Borrego, J. Jr. (2010). Addressing cultural variables in parent training programs with Latino families. Cognitive and Behavioral Practice, 17(2), 157-166. doi:10.1016/j.cbpra.2010.01.002

Barrett, M. S., Chua, W., Crits-Christoph, P., Gibbons, M. B., Casiano, D., \& Thompson, D. (2008). Early withdrawal from mental health treatment: Implications for psychotherapy practice. Psychotherapy, 45(2), 247-267.

Bauermeister, J. J., Canino, G., Bravo, M., Ramírez, R., Jensen, P. S., Chavez, L., ... García, P. (2003). Stimulant and psychosocial treatment of ADHD in Latino/Hispanic children. Journal of the American Academy of Child and Adolescent Psychiatry, 42(7), 851-855. doi:10.1097/01.CHI.0000046864.56865.30

Bird, H. R., Shrout, P. E., Duarte, C. S., Shen, S., Bauermeister, J. J., \& Canino, G. (2008). Longitudinal mental health service and medication use for ADHD among Puerto Rican youth in two contexts. Journal of the American Academy of Child and Adolescent Psychiatry, 47(8), 879-889.

Cabrera, N. J., \& Bradley, R. H. (2012). Latino fathers and their children. Child Development Perspectives, 6(3), 232-238. doi:10.1111/j.1750-8606.2012.00249.x

Calzada, E. J. (2010). Bringing culture into parent training with Latinos. Cognitive and Behavioral Practice, 17(2), 167-175. doi:10.1016/j.cbpra.2010.01.003

Cauce, A. M., Domenech-Rodríguez, M., Paradise, M., Cochran, B. N., Shea, J. M., Srebnik, D., \& Baydar, N. (2002). Cultural and contextual influences in mental health help seeking: A focus on ethnic minority youth. Journal of Consulting and Clinical Psychology, 70(1), 44-55. doi:10.1037//0022-006X.70.1.44

Corkum, P., Bessey, M., McGonnell, M., \& Dorbeck, A. (2015). Barriers to evidence-based treatment for children with attention-deficit/hyperactivity disorder. ADHD Attention Deficit and Hyperactivity Disorders, 7(1), 49-74. doi:10.1007/s12402-014-0152-z

Cuellar, I., Arnold, B., \& Maldonado, R. (1995). Acculturation Rating Scale for Mexican Americans-II: A revision of the original ARSMA scale. Hispanic Journal of Behavioral Sciences, 17(3), 275-304. doi:10.1177/07399863950173001

de Haan, A. M., Boon, A. E., de Jong, J. T., Hoeve, M., \& Vermeiren, R. R. (2013). A meta-analytic review on treatment dropout in child and adolescent outpatient mental health care. Clinical Psychology Review, 33(5), 698-711. doi:10.1016/j.cpr.2013.04.005

Eiraldi, R., \& Diaz, Y. (2010). Use of treatment services for attention-deficit/hyperactivity disorder in Latino children. Current Psychiatry Reports, 12(5), 403-408. doi:10.1007/s11920-010-0139-1

Ennis, S. R., Ríos-Vargas, M., \& Albert, N. G. (2011). The Hispanic population: 2010. Retrieved from https://www.census.gov/prod/cen2010/briefs/c2010br-04.pdf. 
Fernández, M. C., \& Arcia, E. (2004). Disruptive behaviors and maternal responsibility: A complex portrait of stigma, self-blame, and other reactions. Hispanic Journal of Behavioral Sciences, 26(3), 356-372. doi:10.1177/0739986304267208

Forehand, R., \& Kotchick, B. A. (1996). Cultural diversity: A wake-up call for parent training. Behavior Therapy, 27(2), 187-206. doi:10.1016/S0005-7894(96)80014-1

Forehand, R., \& Kotchick, B. A. (2002). Behavioral parent training: Current challenges and potential solutions. Journal of Child and Family Studies, 11(4), 377-384. doi:10.1023/A:1020913422609

García Coll, C. T., \& Marks, A. K. (2012). The immigrant paradox in children and adolescents: Is becoming American a developmental risk? Washington, DC: American Psychological Association.

Garland, A. F., Lau, A. S., Yeh, M., McCabe, K. M., Hough, R. L., \& Landsverk, J. A. (2005). Racial and ethnic differences in utilization of mental health services among high-risk youths. American Journal of Psychiatry, 162(7), 1336-1343. doi:10.1176/appi.ajp.162.7.1336

Gazmararian, J. A., Curran, J. W., Parker, R. M., Bernhardt, J. M., \& DeBuono, B. A. (2005). Public health literacy in America: An ethical imperative. American Journal of Preventive Medicine, 28(3), 317-322. doi:10.1016/j.amepre.2004.11.004

Gerdes, A. C., Kapke, T. L., Grace, M. G., \& Castro, A. (2017). Familias Sanas: A culturally-adapted treatment for Latino youth with ADHD. Manuscript submitted for publication.

Gerdes, A. C., Kapke, T. L., Lawton, K. E., Grace, M., \& Hurtado, G. D. (2015). Culturally adapting parent training for Latino youth with ADHD: Development and pilot. Journal of Latina/o Psychology, 3(2), 71-87. doi:10.1037/lat0000037

Gerdes, A. C., Lawton, K. E., Haack, L. M., \& Hurtado, G. D. (2013). Assessing ADHD in Latino families: Evidence for moving beyond symptomatology. Journal of Attention Disorders, 17(2), 128-140. doi:10.1177/1087054711427396

Gonzales, N. A., Fabrett, F. C., \& Knight, G. P. (2009). Acculturation, enculturation, and the psychosocial adaptation of Latino youth. In F. A. Villarruel, G. Carlo, J. M. Grau, M. Azmitia, N. J. Cabrera, \& T. J. Chahin (Eds.), Handbook of U.S. Latino psychology: Developmental and community-based perspectives (pp. 115-134). Thousand Oaks, CA: SAGE Publications, Inc.

Gopalan, G., Goldstein, L., Klingenstein, K., Sicher, C., Blake, C., \& McKay, M. M. (2010). Engaging families into child mental health treatment: Updates and special considerations. Journal of the Canadian Academy of Child and Adolescent Psychiatry, 19(3), 182-196.

Graetz, B. W., Sawyer, M. G., Baghurst, P., \& Hirte, C. (2006). Gender comparisons of service use among youth with attention-deficit/hyperactivity disorder. Journal of Emotional and Behavioral Disorders, 14(1), 2-11. doi:10.1177/10634266060140010101

Haack, L. M., Gerdes, A. C., Lawton, K. E., \& Schneider, B. W. (2016). Understanding and measuring functional impairment in diverse children with ADHD: Development of the ADHD-FX Scale with an at-risk, community sample. Journal of Attention Disorders, 20(6), 487-500. doi:10.1177/1087054714527791

Haack, L. M., Gonring, K., Harris, M., Gerdes, A. C., \& Pfiffner, L. J. (2019). Assessing impairment in childhood ADHD: Validation of the parent and teacher ADHD-FX rating scale in a dual-site clinical sample. Journal of Attention Disorders, 23(6), 541-552.

Ho, J., Yeh, M., McCabe, K., \& Hough, R. L. (2007). Parental cultural affiliation and youth mental health service use. Journal of Youth and Adolescence, 36(4), 529-542. doi:10.1007/s10964-006-9114-x

Hollingshead, A. A. (1975). Four-factor index of social status. New Haven, CT: Yale University.

Holmbeck, G. N., Li, S. T., Schurman, J. V., Friedman, D., \& Coakley, R. M. (2002). Collecting and managing multisource and multimethod data in studies of pediatric populations. Journal of Pediatric Psychology, 27(1), 5-18. doi:10.1093/jpepsy/27.1.5

Hoza, B., Johnston, C., Pillow, D. R., \& Ascough, J. C. (2006). Predicting treatment response for childhood attention-deficit/hyperactivity disorder: Introduction of a heuristic model to guide research. Applied and Preventive Psychology, 11(4), 215-229. doi:10.1016/j.appsy.2005.11.001 
Ingoldsby, E. M. (2010). Review of interventions to improve family engagement and retention in parent and child mental health programs. Journal of Child and Family Studies, 19(5), 629-645. doi:10.1007/s10826-0099350-2

Kapke, T. L., \& Gerdes, A. C. (2015). Latino family participation in youth mental health services: Treatment retention, engagement, \& response. Clinical Child and Family Psychology Review, 10(4), 329-351. doi:10.1007/s10567-016-0213-2

Kataoka, S. H., Zhang, L., \& Wells, K. B. (2002). Unmet need for mental health care among U.S. children: Variation by ethnicity and insurance status. American Journal of Psychiatry, 159(9), 1548-1555. doi:10.1176/appi.ajp.159.9.1548

Keyes, K. M., Martins, S. S., Hatzenbuehler, M. L., Blanco, C., Bates, L. M., \& Hasin, D. S. (2012). Mental health service utilization for psychiatric disorders among Latinos living in the United States: The role of ethnic subgroup, ethnic identity, and language/social preferences. Social Psychiatry and Psychiatric Epidemiology, 47(3), 383-394. doi:10.1007/s00127-010-0323-y

Kim, R. E., Lau, A. S., \& Chorpita, B. F. (2016). The Impact of Latino caregiver acculturation on treatment engagement in children's community mental health services. Journal of Child and Family Studies, 25(3), 891-901. doi:10.1007/s10826-015-0259-7

Knight, G. P., Gonzales, N. A., Saenz, D. S., Bonds, D. D., Germán, M., Deardorff, J., ... Updegraff, K. A. (2010). The Mexican American Cultural Values Scales for adolescents and adults. Journal of Early Adolescence, 30(3), 444-481. doi:10.1177/0272431609338178

Lara, M., Gamboa, C., Kahramanian, M. I., Morales, L. S., \& Bautista, D. E. (2005). Acculturation and Latino health in the United States: A review of the literature and its sociopolitical context. Annual Review of Public Health, 26(1), 367-397. doi:10.1146/annurev.publhealth.26.021304.144615

Lundahl, B. W., Tollefson, D., Risser, H., \& Lovejoy, M. C. (2008). A meta-analysis of father involvement in parent training. Research on Social Work Practice, 18(2), 97-106. doi:10.1177/1049731507309828

Marín, G. (1992). Issues in the measurement of acculturation among Hispanics. In K. Geisinger (Ed.), Psychological testing of Hispanics (pp. 235-251). Washington, DC: American Psychological Association.

Martinez, R. O., \& Villarruel, F. A. (2009). Preface. In F. A. Villarruel, G. Carlo, J. M. Grau, M. Azmitia, N. J. Cabrera, \& T. J. Chahin (Eds.), Handbook of U.S. Latino psychology: Developmental and community-based perspectives (pp. vii-xi). Thousand Oaks, CA: SAGE Publications, Inc.

McCabe, K. M. (2002a). Factors that predict premature termination among Mexican-American children in outpatient psychotherapy. Journal of Child and Family Studies, 11(3), 347-359.

McCabe, K. M. (2002b). Therapy Attitudes Questionnaire. Unpublished measure available from the author. McCabe, K. M. (2002c). Therapy Expectations Questionnare. Unpublished measure available from the author.

McCabe, K. M., Yeh, M., Garland, A. F., Lau, A. S., \& Chavez, G. (2005). The GANA program: A tailoring approach to adapting Parent Child Interaction Therapy for Mexican Americans. Education and Treatment of Children, 28(2), 111-129.

Miranda, J., Lawson, W., \& Escobar, J. (2002). Ethnic minorities. Mental Health Services Research, 4(4), $231-237$.

Miranda, J., Azocar, F., Organista, K. C., Muñoz, R. F., \& Lieberman, A. (1996). Recruiting and retaining lowincome Latinos in psychotherapy research. Journal of Consulting and Clinical Psychology Review, 64(5), 868-874. doi:10.1037/0022-006X.64.5.868

Morgan, P. L., Hillemeier, M. M., Farkas, G., \& Maczuga, S. (2014). Racial/ethnic disparities in ADHD diagnosis by kindergarten entry. Journal of Child Psychology and Psychiatry, 55(8), 905-913. doi:10.1111/jcpp.12204

Nandi, A., Galea, S., Lopez, G., Nandi, V., Strongarone, S., \& Ompad, D. C. (2008). Access to and use of health services among undocumented Mexican immigrants in a US urban area. American Journal of Public Health, 98(11), 2011-2020. doi:10.2105/AJPH.2006.096222

National Institutes of Health. (2002). Outreach notebook for the inclusion, recruitment and retention of women and minority subjects in clinical research. Retrieved from www4.od.nih.gov/orwh/outreach

Nix, R. L., Bierman, K. L., \& McMahon, R. J. (2009). How attendance and quality of participation affect treatment response to parent management training. Journal of Consulting and Clinical Psychology, 77(3), 429-438. doi:10.1037/a0015028 
Paidpati, C. P., Brawner, B., Eiraldi, R., \& Deatrick, J. A. (2017). Parent and family processes related to ADHD management in ethnically diverse youth. Journal of the American Psychiatric Nurses Association, 23(2), 90-112. doi:10.1177/1078390316687023

Pelham, W. E. J., Gnagy, E. M., Greenslade, K. E., \& Milich, R. (1992). Teacher ratings of DSM-III-R symptoms for the disruptive behavior disorders. Journal of the American Academy of Child and Adolescent Psychiatry, 31(2), 210-218. doi:10.1097/00004583-199203000-00006

Pelham, W. E., Fabiano, G. A., \& Massetti, G. M. (2005). Evidence-based assessment of attention-deficit hyperactivity disorder in children and adolescents. Journal of Clinical Child and Adolescent Psychology, 34(3), 449-476. doi:10.1207/s15374424jccp3403_5

Pelham, W. E., Jr., \& Fabiano, G. A. (2008). Evidence-based psychosocial treatments for attentiondeficit/hyperactivity disorder. Journal of Clinical Child and Adolescent Psychology, 37(1), 184-214. doi:10.1080/15374410701818681

Pew Hispanic Center. (2009). Latino children: A majority are U.S.-born offspring of immigrants. Washington, DC: Pew Research Center.

Pew Hispanic Center. (2011). Census 2010: 50 million Latinos - Hispanics account for more than half of nation's growth in past decade. Washington, DC: Pew Research Center.

Phinney, J. S., \& Ong, A. D. (2007). Conceptualization and measurement of ethnic identity: Current status and future directions. Journal of Counseling Psychology, 54(3), 271-281. doi:10.1037/0022-0167.54.3.271

Quintana, S. M., \& Scull, N. C. (2009). Latino ethnic identity. In F. A. Villarruel, G. Carlo, J. M. Grau, M. Azmitia, N. J. Cabrera, \& T. J. Chahin (Eds.), Handbook of U.S. Latino psychology (pp. 81-98). Thousand Oaks, CA: SAGE Publications, Inc.

Reyno, S. M., \& McGrath, P. J. (2006). Predictors of parent training efficacy for child externalizing behavior problems - A meta-analytic review. Journal of Child Psychology and Psychiatry, 47(1), 99-111. doi:10.1111/j.1469-7610.2005.01544.x

Schwartz, S. J., Unger, J. B., Zamboanga, B. L., \& Szapocznik, J. (2010). Rethinking the concept of acculturation: Implications for theory and research. American Psychologist, 65(4), 237-251. doi:10.1037/a0019330

Steidel, A. G. L., \& Contreras, J. M. (2003). A new familism scale for use with Latino populations. Hispanic Journal of Behavioral Sciences, 25(3), 312-330. doi:10.1177/0739986303256912

Thiruchelvam, D., Charach, A., \& Schachar, R. J. (2001). Moderators and mediators of long-term adherence to stimulant treatment in children with ADHD. Journal of the American Academy of Child and Adolescent Psychiatry, 40(8), 922-928. doi:10.1097/00004583-200108000-00014

U.S. Census Bureau. (2014). Annual estimates of the resident population by sex, age, race, and Hispanic origin for the United States and states: April 1, 2010 to July 1, 2013. Retrieved from http://factfinder.census.gov/faces/tableservices/jsf/pages/productview.xhtml?pid=PEP 2013 PEPASR6 H\&prodType=table

Vega, W. A., Sribney, W. M., Aguilar-Gaxiola, S., \& Kolody, B. (2004). 12- month prevalence of DSM-III-R psychiatric disorders among Mexican Americans: Nativity, social assimilation, and age determinants. Journal of Nervous and Mental Disease, 192(8), 532-541. doi:10.1097/01.nmd.0000135477.57357.b2

Villatoro, A. P., Morales, E. S., \& Mays, V. M. (2014). Family culture in mental health help-seeking and utilization in a nationally representative sample of Latinos in the United States: The NLAAS. American Journal of Orthopsychiatry, 84(4), 353-363. doi:10.1037/h0099844

Yancey, A. K., Ortega, A. N., \& Kumanyika, S. K. (2006). Effective recruitment and retention of minority research participants. Annual Review of Public Health, 27(1), 1-28. doi:10.1146/annurev.publhealth.27.021405.102113 